\title{
A NOTE ON $\frac{4}{n}=\frac{1}{x}+\frac{1}{y}+\frac{1}{z}$
}

\section{XUN QIAN YANG}

ABSTRACr. Denoting by $S(N)$ the number of natural numbers $n$ less than $N$ for which

$$
\frac{4}{n}=\frac{1}{x}+\frac{1}{y}+\frac{1}{z}
$$

has no solutions in positive integers, we show that $S(N) \ll N / \log ^{2} N$.

P. Erdös conjectured that the equation

$$
\frac{4}{n}=\frac{1}{x}+\frac{1}{y}+\frac{1}{z}
$$

has positive integer solutions $x, y, z$ for every natural number $n \geqslant 2$. This problem has attracted the attention of Straus, Bernstein, Shapiro, Oblath and Yamamoto (see [1] for precise references); Chao Ko, Chi Sun and S. J. Chang [2]; and R. W. Jollensten [3]. The best result hitherto obtained is that (1) holds for $n<1.1 \times 10^{7}$.

In [4], W. A. Webb proved that (1) holds for almost all natural numbers. More precisely, let $S(N)$ denote the number of $n$ 's $(n<N)$ for which (1) has no solution. Then

$$
S(N) \ll \frac{N}{\log ^{7 / 4} N} .
$$

Webb's proof is based on Selbeg's sieve method, and is quite complicated. Webb remarked that his technique could be used to improve the exponent from $7 / 4$ to 2 . It is the aim of this note to give a simple proof of this slightly sharper result.

THEOREM A. $S(N) \ll N / \log ^{2} N$.

The proof is based on the following theorem [5, p. 70].

THEOREM B. Let $g$ be a natural number. Let $a_{i}$ and $b_{i}(i=1,2, \ldots, g)$ be pairs of integers satisfying $\left(a_{i}, b_{i}\right)=1(i=1,2, \ldots, g)$ and define

$$
E=\prod_{i=1}^{g} a_{i} \prod_{1<r<s<g}\left(a_{r} b_{s}-a_{s} b_{r}\right) \neq 0 .
$$

Received by the editors March 2, 1981.

1980 Mathematics Subject Classification. Primary 10B25; Secondary 10H30. 
Let $y$ and $x$ be real numbers satisfying $1 \leqslant y \leqslant x$. Further, let $\Re$ be'a set of pitimes for which there exist constants $\delta$ and $A$ such that

$$
\sum_{\substack{p<y \\ p \in \Re_{0}}} \frac{1}{p} \geqslant \delta \log \log y-\ddot{A}
$$

Then

$$
\mid\left\{n: x-y<n \leqslant x,\left(\left(a_{i} n+b_{i}\right), \mathfrak{B}\right)=1 \text {. for } i=1,2, \ldots, g\right\} \mid
$$

$$
\ll \prod_{\substack{p \mid E \\ p \in \Re}}\left(1-\frac{1}{p}\right)^{\rho(p)-g} \frac{y}{\log ^{\delta} g y}
$$

where $\rho(p)$ denotes the number of solutions of

$$
\sum_{i=1}^{g}\left(a_{i} n+b_{i}\right) \equiv 0(\bmod p)
$$

and where the constant implied by the « notation depends on $g$ and $A$ only.

Proof of Theorem A. Obviously,

$$
\frac{4}{n}= \begin{cases}\frac{1}{n(k+1) k}+\frac{1}{n(k+1)}+\frac{1}{v k}, & n=(4 k-1) v, \\ \frac{1}{n k}+\frac{1}{n k v}+\frac{1}{v k}, & n+1=(4 k-1) v, \\ \frac{1}{n k}+\frac{1}{n k(k v-1)}+\frac{1}{k v-1}, & 4 n+1=(4 k-1) v . \\ \frac{1}{n k}+\frac{1}{k(k v-n)}+\frac{1}{n(k v-n)}, & \end{cases}
$$

Hence, if one of the four numbers $n, n+1, n+4,4 n+1$, has a factor of the form $4 k-1$, then (1) holds.

Thus, we may choose

$$
\mathfrak{B}=\{p: p \equiv-1(\bmod 4)\}, \quad y=x, g=4,
$$

and

$$
\prod_{i=1}^{4}\left(a_{i} x+b_{i}\right)=x(x+1)(x+4)(4 x+1)
$$

in Theorem B and obtain,

$$
E=2^{4} \cdot 3^{3} \cdot 5 \neq 0, \quad \rho(3)=2,
$$

and

$$
\prod_{\substack{p \mid E \\ p \in \mathfrak{B}}}\left(1-\frac{1}{p}\right)^{\rho(p)-g}=\left(\frac{2}{3}\right)^{-2}
$$


By Mertens' result [5, p. 35],

$$
\sum_{\substack{p<x \\ p \equiv l(\bmod k)}} \frac{1}{p}=\frac{1}{\varphi(k)} \log \log x+o_{k}(1), \quad(l, k)=1 .
$$

Taking $\delta=\frac{1}{2}$, we obtain Theorem A directly from (2).

\section{REFERENCES}

1. L. J. Mordell, Diophantine equations, Academic Press, New York and London, 1969.

2. Chao Ko, Chi Sun and S. J. Chang, On the equation $4 / n=1 / x+1 / y+1 / z$, J. Sichuan Univ. (Science) 3 (1964).

3. Ralph W. Jollensten, A note on the Egyptian problem, Proceedings of the Seventh Southeastern Conference on Combinatorics, Graphs, and Computing (Louisiana State Univ., Baton Rouge, La., 1976), pp. 351-364.

4. William A. Webb, On $4 / n=1 / x+1 / y+1 / z$, Proc. Amer. Math. Soc. 25 (1970), 578-584. MR 4, 1639.

5. H. Halberstam and H. E. Richert, Sieve methods, Academic Press, New York, 1974.

Department of Mathematics, South West Teacher's College, Chongqing, Sichuan, People's Republic OF ChINA 